\title{
CONFÉRENCE INTERNATIONALE SUR L'HISTOIRE DE LA PSYCHOLOGIE SOCIALE
}

\author{
LES 18, 19 ET 20 AVRIL 1991
}

\author{
à l'Institut pour la Recherche sur les sociétés contemporaines \\ (I.R.E.S.C.O.-C.N.R.S.) 59-61, rue Pouchet, 75849 Paris (17 $)$, \\ Salle de conférence, rez-de-chaussée.
}

Colloque organisé conjointement par la Section de psychologie sociale de la Société française de psychologie, la Société française pour l'histoire des sciences de l'homme, la Section d'histoire et de philosophie de la Société canadienne de psychologie et Cheiron : International Society for the History of the Behavioral and Social Sciences.

\section{Thème et organisation du colloque :}

La Psychologie sociale a représenté des champs de pratiques et de recherche qui ont nécessairement varié au cours de l'histoire. L'histoire de la Psychologie sociale pourra être envisagée comme une réflexion sur l'évolution de la pensée systématique/scientifique relative aux objets psychosociaux ; réflexion qui contribuera à délimiter à nouveau le champ de la discipline par la mise en perspective des thèmes parfois divergents qui se sont emparés à un moment ou à un autre des questions psychosociales. Parmi les questions qui peuvent contribuer à cette mise en perspective historique (et prospective), le programme des communications aborde entre autres les thèmes suivants :

Comment certains grands problèmes psychosociaux comme les problématiques du $\mathrm{XIX}^{\mathrm{e}}$ siècle ( questions sociales ", psychologie des foules, de l'identité et du nationalisme) et les problèmes sociaux du $\mathrm{xx}^{\mathrm{e}}$ siècle (racisme, conformisme, influence sociale, communication et relations interpersonnelles ou de groupe(s)) ont évolué, disparu, migré vers d'autres disciplines ou ont fait des emprunts interdisciplinaires?

Comment la Psychologie sociale a évolué soit comme recherche soit comme pratique sociale en fonction des influences institutionnelles et des courants idéologiques et scientifiques?

Les contributeurs appartiennent à ce que nous appelons aujourd'hui Psychologie sociale mais proviennent également d'autres disciplines (historiens, sociologues, archivistes, etc.) dont les travaux s'articulent avec la Psychologie sociale ou traitent

Revue de synthèse: IV S. N 4, oct.-déc. 1990. 
de l'histoire des thèmes et objets psychosociaux. A la fin des présentations diverses sur l'histoire de la Psychologie sociale, une séance sera réservée à une discussion de synthèse tirant les enseignements de ce passé pour une réflexion sur l'état actuel de la discipline et sur une vision de son avenir, en particulier en France.

Les langues envisagées pour les communications sont le frangais et l'anglais.

\section{Le comité d'organisation :}

Section de Psychologie sociale de la Société française de psychologie : A. Blanchet, A. Doma, J. M. Jakobi,

Société française pour l'histoire des sciences de l'homme : B. Matalon,

Section d'histoire et de philosophie de la Société canadienne de psychologie :

\section{Lubek,}

Cheiron (International Society for the History of the Behavioral and Social Sciences) : H. Minton.

Avec l'aide amicale de G. Fourcade (S.F.P.) et de G. McGuire (S.C.P.) et avec le soutien de la Direction de l'I.R.E.S.C.O.. 